\title{
THE IMPACT OF FIRMS' CHARACTERISTICS ON EXPORT BARRIERS' PERCEPTION: A CASE OF SERBIAN EXPORTERS
}

\author{
Predrag D. Radojevic, Darko Marjanovic, Tatjana Radovanov*
}

\begin{abstract}
:
This papers analyses impact of firm characteristics, i.e. size, length of exporting experience, capital ownership and type of industry on export barriers' perception in case of Serbian exporters. This study is aimed at an identification of the barriers to export among examined factors of firm's internal environment, domestic business environment and foreign markets to rank barriers according to their level of impact, to spot differences in evaluation of barriers depending on firm's characteristics, and to examine correlation between firm's characteristics and barriers to export. Main hypothesis in this research was that barriers to export for Serbian exporters have been similar to those faced by exporters in other countries, and that the level of their influence depends on firm's characteristics. The empirical research has been conducted through a survey, using a questionnaire with 178 exporters taking part in it. Collected data have been analysed by descriptive statistics, differences among groups and correlation tests. The results imply that most export barriers refer to domestic business environment and that there is a correlation among firm's size, length of export experience and capital ownership with certain factors that may cause problems for exporting business.
\end{abstract}

Keywords: export, firm's characteristics, factors of internal environment factors, factors of domestic business environment, factors of foreign markets, Serbia

JEL Classification: F23, F14, M16

\section{Introduction}

After more than a decade of transition Serbia failed to significantly increase its export, make it more competitive and improve its structure (The World Bank, 2011). Some of the latest documents and strategies for economic recovery of Serbia underline importance of export growth for Serbian economy and recommend the increase of its share in gross domestic product to at least 50\%. For achieving this result it needs an average annual export growth rate of at least $13.5 \%$ (Foundation for the Advancement of Economics, 2010; Petrovic, 2011).

Predrag D. Radojevic, University of Belgrade, Faculty of Economics, Serbia, PhD. student (predrag. radojevic@open.telekom.rs); Darko Marjanovic, University of Novi Sad, Faculty of Economics in Subotica, Serbia, PhD student; Tatjana Radovanov, KDI School for Public Policy and Management, Seoul, South Korea, MSc student.

Authors would like to express special thanks to Economic Department of Czech Republic Embassy in Serbia, Czech Trade Office in Belgrade and SIEPA for their help.

We dedicated this paper to our colleague Ana Jelaca Jasko, MA (1980-2012). She was PhD student at European Center for Peace and Development of the United Nations University and Research Associate at the Institute for Economic Sciences in Belgrade. 
This paper was led by a notion that Serbian export cannot be enhanced without examining exporters' views. Its purpose is to research impact of certain firm characteristics, i.e. firm's size, length of export experience, capital ownership and type of the industry on export barriers perception. This topic have often been analysed and researched in literature (Zou and Stan, 1998; Tesform and Lutz, 2006; Sousa et al., 2008; Cole, et al., 2010). But, it has still not been sufficiently researched when it comes to small, less developed countries, such as Serbia. Also, the lack of agreement among researchers of the size and direction of the impact of certain firm's characteristics on export barriers' perception makes this theme actual.

Aim of this paper was manifold. Firstly, to identify which factors among examined may represent a barrier for Serbian exporters and ranking export barriers by the level of their influence. Secondly, to spot differences in impact of export barriers to different groups of exporters classified according to their previously mentioned characteristics. Thirdly, to examine correlations between firm's characteristics and export barriers' perception. Purpose of the listed aims was to obtain information on the direction, in which it could be directed export policy in Serbia and in which way firm's management may reorganize the business process in exporting firms. In addition, this paper tries to contribute to the discussion on firm's characteristics and export barriers' perception.

To achieve the stated purpose and aims this paper is elaborated as follows. First, there is a brief literature review about the firm's characteristics and the various factors that can create barriers to exports are added. It gave possibility for research conceptual framework for empiric research building and for hypotheses set up. After that follows the methodology. Then, the research results are presented. The paper ends with a discussion and conclusion with research findings and implications for economy policy makers, firm's management and future researches.

\section{Literature Review}

Numerous studies discuss export issues from exporters' viewpoints and present different findings in relation to the firm's characteristics and export barriers perception, and a number of factors have been spotted that may become barriers to the exporting business.

The relationship between firm's size and exports is often considered as being a positive one, i.e. the export of large firms is the less affected by factors that can create barriers to exports in relation to small and medium (Majocchi et al., 2005; Jauhari, 2007). But, there are studies that oppose this finding, or fail to find the link (Haahti et al., 2005; Pla and Alegre, 2007). There are also different results pertaining to influence of length of export experience to export. One shows that barriers to export have less impact on more experienced firms (Suarez-Ortega, 2003; Stoian et al., 2011). Others cannot find link, or show that firms with shorter export experience may be more successful in comparison to older exporters (Cavusgil, 1994; Dominquez and Sequeira, 1993; Moen and Servais, 2002; Lefebvre and Lefebvre, 2001). Studies dealing with impact of 
capital ownership on export are typical for developing countries and differentiate firms with domestic and dominant domestic capital and firms with foreign and dominant foreign capital. Nonetheless, their findings differ as well. One claim that firms with foreign and dominant foreign capital have better prospects export wise (Cole et al., 2010; Filatotchev et al., 2008). The others prove that firms with domestic capital might be more successful in export than firms with foreign capital (Sharma, 2003; Jenkins, 1979). Type of industry where the firm belongs, in terms of technical and technological level of production process, may also affect export. Some studies underscore that high technological firms might be more capable of overcoming barriers to export (Wheeler et al., 2008; Lopez and García, 2005). On the other hand, there are studies the results of which oppose these findings or fail to find the link between them, and emphasise that firms may differ within the very same industry branch and not only among different industries if we contrast them against the level of this firm characteristic (Gao et al., 2010; Lefebrve and Lefebrve, 2001).

There are many classifications of factors that may look as barriers to export in the literature (Arteaga-Ortiz and Fernandez-Ortiz, 2010). According to some authors, there are external factors, deriving outside of the firm, and internal factors stemming from the inside of the firm (Sousa et al., 2008). Others divide them into four groups - factors related to knowledge, internal firm resources, procedures and foreign markets (Ramaswami and Yang, 1990; Lages, 2000; Kneller \& Pisu, 2011). Third groups of authors underline that export of firm is influenced by internal forces stemming from characteristics of the firm and its production program, and external force formed by characteristics for industrial branch this firm belongs to and characteristics of foreign markets the firm has been exporting to (Cavusgil and Zou, 1994). These two groups of forces produce four groups of factors that may have an impact on export, such as external, operational, internal and informational factors (Katsikeas and Morgan, 1994; Tesfom and Lutz, 2006; Neupert et al., 2006).

\section{Research Conceptual Framework and Hypotheses}

Purpose of the literature review on relation between certain firm's characteristics and factors that may pose export barriers has been twofold. Firstly, it was a base for research conceptual framework for the empirical research building. Secondly, main and individual hypotheses that should have been tested through empiric research have been formulated on it.

A list of factors that may pose barriers to export from previous studies has been mainly followed up in this paper. But, few new factors have been added, which authors of this paper deemed to be valuable enough to examine with respect to specificities of Serbian exporters. This refers to national export branding policy in Serbia, since there is no internationally recognised Serbian export brand, as well as exporters' associations issues, since process of clustering has just started in the domestic economy. Unlike previous division of factors that may pose export barriers, this paper has taken environment 
factors derive from as its classification criterion. Accordingly, as presented in Figure 1, factors that may pose export barriers have been divided into factors of firm internal environment, domestic business environment and foreign market.

Figure 1

Research Conceptual Framework

\section{FACTORS}

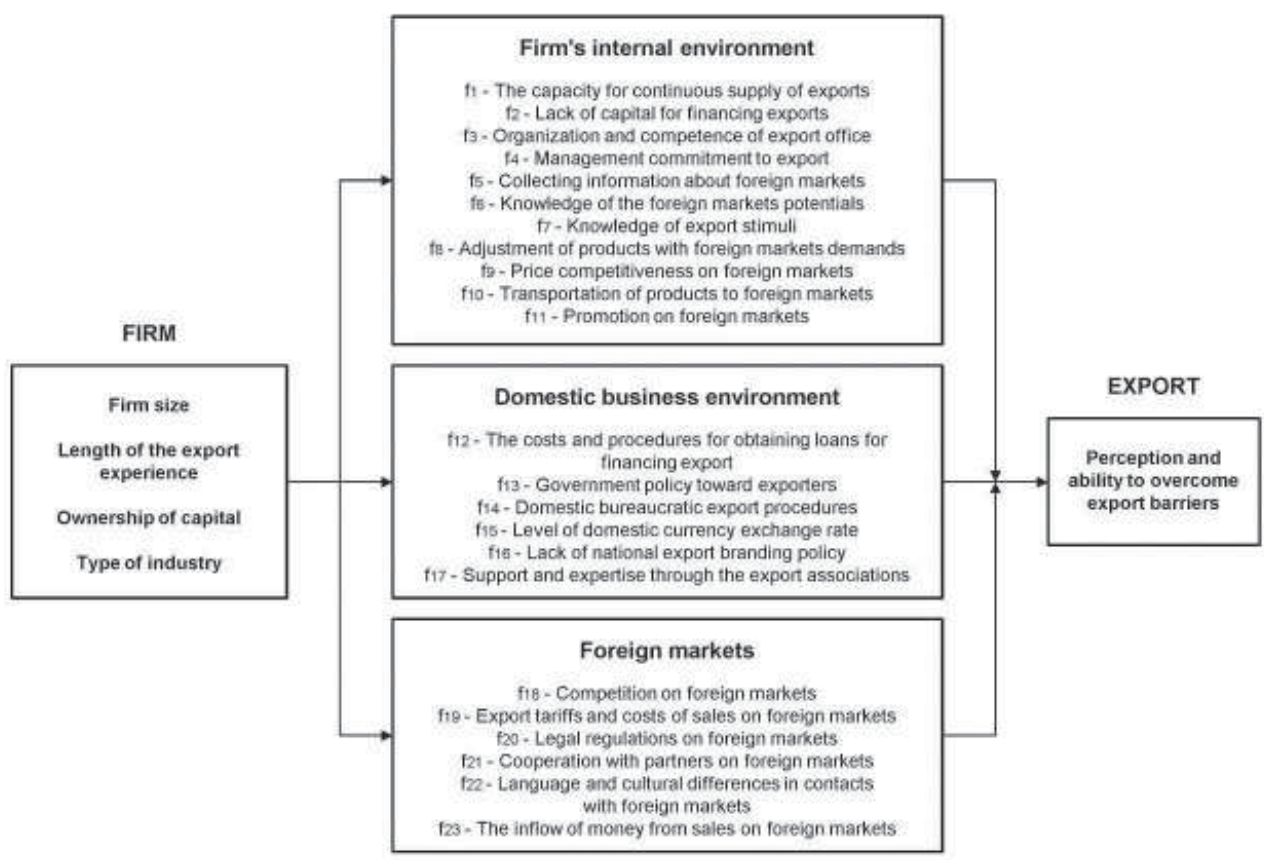

Source: authors

Research hypotheses derive from the literature review and presented research conceptual framework. Assuming that Serbian exporters have been influenced by factors causing export barriers similar to those that have impact on exporters in other countries, firm perception of export barriers and firm ability to overcome them depend on firm characteristics:

Hypotheses 1: Firm size positive affects export.

Hypotheses 2: Length of export business positive affects export.

Hypotheses 3: Dominant presence of foreign capital in firm positive affects export.

Hypotheses 4: Firm belonging to high technological industries positive affects export. 


\section{Methodology of the Study}

\section{Research design}

The empirical research on impact of firm characteristics on export business barriers perception was conducted through a survey. Survey questionnaire had two parts. The first one was composed of data on size, length of export experience, ownership of capital and type of industry firms belong to. Independent variables were formed on the basis of these data. The second part included 23 factors of firm's internal environment, domestic business environment and foreign markets that may pose barriers to export. Dependent variables were formed on the basis of these data. Respondents were assessing factors on the scale ranging from 1 to 5 depending on the level of influence on export (point $1=$ "no influence at all", point $5=$ "fully influenced"). Only factors with average grade higher than 2.5 have been considered to be barriers to export.

The basis for research formed of 552 Serbian manufacture firms with export income of at least one million USD in 2011 according to the Serbia Investment and Export Promotion Agency data. Questionnaires were sent to respondents via e-mails. All firms from the basis were included in the research, having in mind type of delivery of questionnaires and size of the basis.

\section{Testing research instrument}

Validity of the questionnaire was tested at two levels. Firstly, positive assessment was obtained from experts in Serbia Investment and Export Promotion Agency. Secondly, the questionnaire was tested by 20 firms form the basis, which published to be assisting academic research activities on their website. Upon receipt of answers, minor amendments have been made.

\section{Data collection}

The research was carried out in the course of March and April, 2012. Respondents were first sent e-mail with the questionnaire attached, followed by four further follows-ups. In total 204 firms sent completed questionnaires, out of which 178 were correctly filled-in, whereas 26 were not. Hence the respond rate in this research was 32.25\%. This considered satisfactory since, according to some findings, average respond rate in research involving business entities is around 21\% (Dillman, 2007, p. 323). Main data on firms taking part in the research are presented in the Table 1.

Internal consistency and reliably of answers measured by Cronbach's alpha coefficient was 0.806, which indicated good reliability (DeVellis, 2003, p. 90). 
Table 1

Characteristics of Enterprises Taking Part in the Research

\begin{tabular}{|l|c|r|l|c|c|}
\hline Firm's size & Frequency & $\%$ & Capital ownership & Frequency & $\%$ \\
\hline Small (S) & 26 & 14.6 & Domestic (D) & 117 & 65.7 \\
\hline Medium (M) & 98 & 55.1 & Foreign (F) & 61 & 34.3 \\
\hline Large (L) & 54 & 30.3 & & Frequency & $\%$ \\
\hline Export expirience & Frequency & $\%$ & Type of industry & 6 & 2.8 \\
\hline Up to 5 years (<5) & 16 & 9 & High-technology (HTI) & 30 & 16.3 \\
\hline $\begin{array}{l}\text { From 6 to 20 years } \\
(6-20)\end{array}$ & 71 & 39.9 & $\begin{array}{l}\text { Medium-high-technology } \\
(\text { MHTI) }\end{array}$ & 58 & 33.7 \\
\hline $\begin{array}{l}\text { From 21 to 50 years } \\
(21-50)\end{array}$ & 51 & 28.7 & $\begin{array}{l}\text { Medium-low-technology } \\
(\text { MLTI) }\end{array}$ & 84 & 47.2 \\
\hline Over 50 years $(>50)$ & 40 & 22.5 & Low-technology (LTI) & & \\
\hline
\end{tabular}

Source: authors' research

\section{Checking reasons for non-participation in the research}

Several t-tests according the literature have been conducted in order to establish whether it is likely that views of firms taking part in the research may differ significantly from those that failed to reply (Armstrong and Overton, 1977; Weisberg, 2005, pp. 159-204). Those tests have not discovered significant differences. In the end, through telephone interviews with a random sample of 20 firms that did not take part in the research were checked reasons for non-participation. According to the answers, $35 \%$ of firms did not participate since it was not in line with their business policy, in 30\% of firms' export managers did not have time, $15 \%$ of firms were not given permission to take part by their head companies, in 5\% of firms' export managers were away and in $15 \%$ of firms have not been possible to get any answer.

\section{Data processing and analysis}

For data process and analyses there were used descriptive statistics techniques. Existence of statistically significant differences in evaluation of investigated factors among different groups of firms sorted by their characteristics was checked by the one way analysis of variance of different groups (ANOVA) and the Kruskal-Wallis test (K-W test), when there were three and more groups in independent variable, and independent sample t-test and Mann-Whitney U tests (M-V U test), when there were two groups within independent variable. Both types of statistic tests have been used to ensure that the results were not the result of an inherent mathematical bias of parametric and nonparametric statistical analysis techniques. The Spearman's rank correlation was used to check links among independent variables, i.e. firm characteristics and dependent variables, i.e. factors of firm internal environment, domestic business environment and foreign markets. Values obtained by analysis of differences between groups and correlation were assessed by Cohen's criteria (Ellis, 2010, pp. 40-42). 


\section{Research Findings}

\section{Influence of factors of firm's internal environment, domestic business environment and foreign markets}

Average scores for investigated factors that may pose barriers to export are shown in Table 2:

1) Within factors of firm internal environment the biggest barriers are lack of capital for export financing and achieving price competitiveness on foreign markets. Other barriers include foreign market promotion, collection of information about foreign markets and adjustment of products to foreign markets requirements. Other factors, according to results, are not barriers to export;

2) Within factors of domestic business environment all investigated factors were grouped as barriers. The largest barrier is expense and procedures for granting of loans for export financing. Second is domestic currency exchange rate, followed by lack of national export branding policy, government policy towards exporters, nonexistence of exporters associations and local bureaucratic export procedures;

3) Within factors of foreign markets only one could be considered as a significant barrier and that is competition. Other identified barriers, but with much lower average scores, includes high export tariffs and legal regulation.

Table 2

Average Evaluation of Influence of Investigated Factors

\begin{tabular}{|c|c|c|c|c|c|c|c|c|}
\hline \multirow{2}{*}{ Environment } & \multirow{2}{*}{ Factors } & \multicolumn{5}{|c|}{ Percents } & \multirow[t]{2}{*}{ Mean * } & \multirow{2}{*}{ St. Deviation } \\
\hline & & 1 & 2 & 3 & 4 & 5 & & \\
\hline \multirow{11}{*}{$\begin{array}{l}\text { Firm's internal } \\
\text { environment }\end{array}$} & f 1 & 31.5 & 32 & 20.2 & 13.5 & 2.8 & 2.24 & 1.12 \\
\hline & f 2 & 9.6 & 10.7 & 33.1 & 27.5 & 19.1 & 3.36 & 1.89 \\
\hline & f3 & 47.8 & 29.2 & 18 & 3.9 & 1.1 & 1.81 & 0.94 \\
\hline & f 4 & 48.9 & 25.8 & 15.2 & 7.9 & 2.2 & 1.89 & 1.07 \\
\hline & f5 & 12.9 & 21.9 & 39.3 & 19.7 & 6.2 & 2.84 & 1.08 \\
\hline & $f 6$ & 23 & 28.7 & 30.9 & 14 & 3.4 & 2.46 & 1.09 \\
\hline & $\mathrm{f} 7$ & 39.9 & 24.2 & 25.8 & 8.4 & 1.7 & 2.08 & 1.07 \\
\hline & f 8 & 16.9 & 27 & 38.2 & 14 & 3.9 & 2.61 & 1.05 \\
\hline & $f 9$ & 10.7 & 14.6 & 39.3 & 24.7 & 10.7 & 3.10 & 1.11 \\
\hline & $f 10$ & 29.2 & 27.5 & 25.8 & 11.8 & 5.6 & 2.37 & 1.18 \\
\hline & $f 11$ & 17.4 & 19.7 & 32 & 18.5 & 12.4 & 2.89 & 1.26 \\
\hline \multirow{6}{*}{$\begin{array}{l}\text { Domestic business } \\
\text { environment }\end{array}$} & $f 12$ & 1.1 & 1.7 & 29.2 & 41 & 27 & 3.93 & 0.83 \\
\hline & $f 13$ & 6.2 & 3.9 & 41 & 27 & 21.9 & 3.54 & 1.07 \\
\hline & $\mathrm{f} 14$ & 3.4 & 6.2 & 60.7 & 18.5 & 11.2 & 3.28 & 0.87 \\
\hline & $f 15$ & 1.1 & 3.4 & 34.3 & 35.4 & 25.8 & 3.81 & 0.90 \\
\hline & $f 16$ & 0.6 & 1.7 & 47.8 & 28.7 & 28.7 & 3.69 & 0.85 \\
\hline & $f 17$ & 0.6 & 7.9 & 65.2 & 15.2 & 11.2 & 3.29 & 0.79 \\
\hline \multirow[t]{6}{*}{ Foreign markets } & $f 18$ & 3.9 & 5.1 & 13.5 & 33.1 & 44.4 & 4.09 & 1.06 \\
\hline & $f 19$ & 14 & 30.3 & 34.3 & 14.6 & 6.7 & 2.70 & 1.09 \\
\hline & $f 20$ & 14 & 32 & 34.8 & 9.6 & 9.6 & 2.69 & 1.13 \\
\hline & f 21 & 29.2 & 36.5 & 25.8 & 7.3 & 1.1 & 2.15 & 0.96 \\
\hline & f 22 & 59.6 & 24.7 & 11.8 & 2.8 & 1.1 & 1.61 & 0.88 \\
\hline & f 23 & 25.8 & 32.6 & 28.1 & 8.4 & 5.1 & 2.34 & 1.10 \\
\hline
\end{tabular}

* Factor is a barrier to export for Mean $>2.5$

Source: authors' research 


\section{Differences in evaluation of internal environment factors, domestic business environment and foreign markets by firm characteristics}

The ANOVA and K-W test results, which have been presented in Table 3, find existence of three statistically significant differences in evaluation of investigated factors depending on the firm size among:

1) Small and large firms in assessing influence of lack of capital for financing export.

2) Small and large firms in assessing influence of domestic currency exchange rate.

3) Medium and large firms in assessing the impact of legal regulation on foreign markets.

Table 3

Differences in Evaluation of Investigated Factors by the Firms' Size

\begin{tabular}{|c|c|c|c|c|c|c|c|c|}
\hline \multirow{3}{*}{ Environment } & \multirow{3}{*}{ Factors } & \multicolumn{3}{|c|}{ Mean (Standard Deviation) } & \multirow{2}{*}{\multicolumn{2}{|c|}{ ANOVA }} & \multirow{2}{*}{\multicolumn{2}{|c|}{ K-W test }} \\
\hline & & \multicolumn{3}{|c|}{ Firm size } & & & & \\
\hline & & $S, N=26$ & $M, N=98$ & L, $N=54$ & $\mathbf{F}$ & $\mathbf{p}^{*}$ & $x^{2}$ & $\mathbf{p}^{*}$ \\
\hline \multirow{11}{*}{$\begin{array}{l}\text { Firm's } \\
\text { internal } \\
\text { environment }\end{array}$} & f 1 & $2.42(1.362)$ & $2.26(1.087)$ & $2.13(1.065)$ & 0.614 & 0.542 & 0.731 & 0.694 \\
\hline & f 2 & $3.81(1.096)$ & $3.38(1.180)$ & $3.11(1.192)$ & 3.125 & 0.046 & 5.990 & 0.049 \\
\hline & f 3 & $1.77(0.815)$ & $1.89(1.034)$ & $1.70(0.816)$ & 0.698 & 0.499 & 0.609 & 0.738 \\
\hline & f 4 & $2.12(1.211)$ & $1.86(1.015)$ & $1.83(1.112)$ & 0.692 & 0.502 & 1.063 & 0.588 \\
\hline & f 5 & $3.00(0.980)$ & $2.74(1.143)$ & $2.94(0.998)$ & 0.920 & 0.400 & 1.485 & 0.476 \\
\hline & f 6 & $2.88(0.993)$ & $2.42(1.130)$ & $2.33(1.046)$ & 2.425 & 0.091 & 5.045 & 0.080 \\
\hline & $\mathrm{f} 7$ & $2.54(1.140)$ & $1.98(0.995)$ & $2.04(1.132)$ & 2.920 & 0.057 & 5.170 & 0.075 \\
\hline & f 8 & 2.58 (1.137) & $2.54(1.027)$ & $2.76(1.045)$ & 0.772 & 0.464 & 1.492 & 0.474 \\
\hline & f 9 & $2.96(1.248)$ & $3.02(1.035)$ & $3.31(1.179)$ & 1.459 & 0.235 & 2.720 & 0.257 \\
\hline & f 10 & $2.58(1.301)$ & $2.32(1.181)$ & $2.37(1.138)$ & 0.496 & 0.610 & 0.845 & 0.655 \\
\hline & $\mathrm{f} 11$ & $2.92(1.383)$ & $2.81(1.249)$ & $3.02(1.205)$ & 0.510 & 0.602 & 0.942 & 0.624 \\
\hline \multirow{6}{*}{$\begin{array}{l}\text { Domestic } \\
\text { business } \\
\text { environment }\end{array}$} & f 12 & $4.08(0.935)$ & $3.96(0.872)$ & $3.74(0.757)$ & 1.741 & 0.178 & 5.488 & 0.064 \\
\hline & f 13 & $3.69(1.320)$ & $3.56(1.066)$ & $3.44(0.945)$ & 0.495 & 0.611 & 1.645 & 0.439 \\
\hline & f 14 & $3.27(1.116)$ & $3.35(0.744)$ & $3.17(0.746)$ & 0.748 & 0.475 & 1.213 & 0.545 \\
\hline & f 15 & $4.12(0.864)$ & $3.87(0.893)$ & $3.57(0.882)$ & 3.668 & 0.028 & 7.108 & 0.029 \\
\hline & f 16 & $3.73(0.827)$ & $3.74(0.877)$ & $3.56(0.793)$ & 0.917 & 0.402 & 1.278 & 0.528 \\
\hline & $f 17$ & $3.42(0.758)$ & $3.36(0.815)$ & $3.09(0.734)$ & 2.448 & 0.089 & 6.072 & 0.058 \\
\hline \multirow{6}{*}{$\begin{array}{l}\text { Foreign } \\
\text { markets }\end{array}$} & $f 18$ & $3.92(0.977)$ & $4.08(1.109)$ & $4.19(1.029)$ & 0.586 & 0.349 & 2.104 & 0.349 \\
\hline & $f 19$ & $2.88(1.306)$ & $2.55(1.047)$ & $2.87(1.047)$ & 0.145 & 0.124 & 4.175 & 0.124 \\
\hline & $\mathrm{f} 20$ & $2.77(1.032)$ & $2.49(1.086)$ & $3.00(1.182)$ & 0.025 & 0.015 & 8.465 & 0.015 \\
\hline & $\mathrm{f} 21$ & $1.92(0.845)$ & $2.15(0.945)$ & $2.24(1.045)$ & 0.385 & 0.438 & 1.653 & 0.438 \\
\hline & f 22 & $1.65(1.018)$ & $1.65(0.921)$ & $1.52(0.746)$ & 0.648 & 0.842 & 0.344 & 0.842 \\
\hline & f 23 & $2.42(0.945)$ & $2.26(1.106)$ & $2.46(1.177)$ & 0.501 & 0.459 & 1.558 & 0.459 \\
\hline
\end{tabular}

* Statistically significant at $p<.05$

Source: authors' research 
The ANOVA and K-W test results, of which are shown in Table 4, find the five statistically significant differences in evaluation of investigated factors depending on the length of export experience of the firm among:

1) Firms with shorter than 5 years and firms with export experience between 21 and 50 years in estimation of the impact of knowledge on export incentive;

2) Firms with exporting more than 50 years on one hand, and firms with exports between 6 and 20 years, and firms which export between 21 and 50 years on the other, in estimation of the impact of adjustment of the products to the foreign markets requirements;

3) Firms with exporting more than 50 years and firms which export between 6 and 20 years in estimation of the impact of achieving price competitiveness;

4) Firms with exporting more than 50 years on one hand and the firms with export experience between 6 and 20 years and between 21 and 50 years on the other in estimation of the impact of the level of export tariffs;

5) Firms with exporting more than 50 years on one hand and all three other groups of firms on the other in estimation of the impact of legal regulation on foreign markets.

Table 4

Difference in the Estimation of the Investigated Factors by the Firms' Export Experience

\begin{tabular}{|c|c|c|c|c|c|c|c|c|c|}
\hline \multirow{3}{*}{$\begin{array}{l}\text { Environ- } \\
\text { ment }\end{array}$} & \multirow{3}{*}{$\begin{array}{l}\text { Fac- } \\
\text { tors }\end{array}$} & \multirow{2}{*}{\multicolumn{4}{|c|}{ Mean (Standard Deviation) }} & \multirow{2}{*}{\multicolumn{2}{|c|}{ ANOVA }} & \multirow{2}{*}{\multicolumn{2}{|c|}{ K-W test }} \\
\hline & & & Length of the & & & & & & \\
\hline & & $<5, N=16$ & $6-20, N=71$ & $21-50, N=51$ & $>50, N=40$ & $\mathbf{F}$ & $\mathbf{p}^{*}$ & $x^{2}$ & $\mathbf{p}^{*}$ \\
\hline \multirow{11}{*}{$\begin{array}{l}\text { Firm's } \\
\text { internal } \\
\text { environ- } \\
\text { ment }\end{array}$} & f1 & $1.88(1.147)$ & $2.34(1.195)$ & $2.10(1.153)$ & $2.40(0.900)$ & 1.296 & 0.277 & 5.445 & 0.142 \\
\hline & f2 & $3.06(1.436)$ & $3.38(1.258)$ & $3.22(1.189)$ & $3.62(0.897)$ & 1.265 & 0.288 & 2.750 & 0.432 \\
\hline & f3 & $1.56(0.629)$ & $1.86(0.975)$ & $1.71(0.965)$ & $1.98(0.947)$ & 1.050 & 0.372 & 3.417 & 0.332 \\
\hline & $\mathrm{f} 4$ & $1.75(0.931)$ & $2.08(1.204)$ & $1.71(1.026)$ & $1.82(0.903)$ & 1.428 & 0.236 & 3.671 & 0.299 \\
\hline & f5 & $2.62(1.204)$ & $2.79(1.081)$ & $2.82(1.126)$ & $3.05(0.959)$ & 0.773 & 0.511 & 2.535 & 0.469 \\
\hline & f6 & $2.38(1.147)$ & $2.52(1.012)$ & $2.31(1.122)$ & $2.58(1.196)$ & 0.552 & 0.647 & 1.781 & 0.619 \\
\hline & $\mathrm{f7}$ & $2.38(1.258)$ & $2.20(1.090)$ & $1.73(0.940)$ & $2.20(1.043)$ & 2.803 & 0.041 & 8.191 & 0.042 \\
\hline & f8 & $2.62(1.258)$ & $2.44(0.937)$ & $2.47(1.065)$ & $3.10(1.008)$ & 4.068 & 0.008 & 9.992 & 0.019 \\
\hline & f9 & $2.94(1.237)$ & $2.87(1.068)$ & $3.14(1.200)$ & $3.52(0.933)$ & 3.158 & 0.026 & 9.364 & 0.025 \\
\hline & $\mathrm{f} 10$ & $2.31(1.078)$ & $2.30(1.164)$ & $2.22(1.172)$ & $2.72(1.240)$ & 1.613 & 0.188 & 4.492 & 0.213 \\
\hline & $\mathrm{f} 11$ & $2.62(1.455)$ & $2.80(1.142)$ & $2.80(1.327)$ & $3.25(1.235)$ & 1.549 & 0.204 & 4.092 & 0.252 \\
\hline \multirow{6}{*}{$\begin{array}{l}\text { Domestic } \\
\text { business } \\
\text { environ- } \\
\text { ment }\end{array}$} & f12 & $4.06(0.680)$ & $3.83(0.926)$ & $4.04(0.916)$ & $3.82(0.675)$ & 0.896 & 0.444 & 3.430 & 0.330 \\
\hline & $\mathrm{f} 13$ & $3.31(1.078)$ & $3.65(1.030)$ & 3.45 (1.189) & $3.58(0.984)$ & 0.610 & 0.609 & 1.510 & 0.680 \\
\hline & $\mathrm{f} 14$ & $2.94(0.772)$ & $3.39(0.933)$ & $3.18(0.865)$ & $3.35(0.770)$ & 1.578 & 0.196 & 4.855 & 0.183 \\
\hline & f15 & $3.56(0.892)$ & $3.75(0.906)$ & $4.02(0.969)$ & $3.78(0.768)$ & 1.479 & 0.222 & 5.173 & 0.160 \\
\hline & f16 & $3.81(0.834)$ & $3.59(0.888)$ & $3.80(0.775)$ & $3.65(0.864)$ & 0.767 & 0.514 & 2.800 & 0.423 \\
\hline & $\mathrm{f} 17$ & $3.50(0.894)$ & $3.24(0.686)$ & $3.45(0.923)$ & $3.08(0.694)$ & 2.212 & 0.088 & 6.787 & 0.079 \\
\hline \multirow{6}{*}{$\begin{array}{l}\text { Foreign } \\
\text { markets }\end{array}$} & $\mathrm{f} 18$ & 3.81 (1.167) & $4.01(1.189)$ & $4.14(1.059)$ & $4.28(0.751)$ & 0.918 & 0.434 & 1.785 & 0.618 \\
\hline & f19 & $2.62(1.088)$ & 2.59 (1.103) & $2.51(0.987)$ & $3.15(1.122)$ & 3.140 & 0.027 & 8.022 & 0.046 \\
\hline & $\mathrm{f} 20$ & $2.50(0.894)$ & $2.55(1.066)$ & $2.57(1.204)$ & $3.15(1.122)$ & 3.046 & 0.030 & 8.799 & 0.032 \\
\hline & f21 & $2.12(0.885)$ & $2.14(0.930)$ & $2.00(0.959)$ & $2.35(1.051)$ & 0.992 & 0.398 & 3.105 & 0.376 \\
\hline & f22 & $1.88(1.204)$ & $1.58(0.822)$ & $1.47(0.809)$ & $1.75(0.927)$ & 1.275 & 0.285 & 3.035 & 0.386 \\
\hline & f23 & $2.62(1.310)$ & $2.23(1.045)$ & $2.27(0.961)$ & $2.52(1.281)$ & 1.044 & 0.375 & 2.125 & 0.547 \\
\hline
\end{tabular}

* Statistically significant at $p<.05$

Source: authors' research 
Independent sample t-test and the M-W U test, whose results are shown in Table 5, confirm the existence of the seven statistically significant differences in estimation of the investigated factors among firms with domestic and firms with the foreign capital, regarding estimation of impact of:

1) Lack of the capital for financing exports;

2) Commitment of the management to export business;

3) Collecting information on foreign markets;

4) Promotion on foreign markets;

5) Lack of national exports branding policy;

6) Cooperation with the partners from foreign markets;

7) Money inflow from the sale on foreign markets.

Table 5

Differences in Estimation of the Investigated Factors by the Firms ‘ Capital

\begin{tabular}{|c|c|c|c|c|c|c|c|c|}
\hline \multirow{3}{*}{ Environment } & \multirow{3}{*}{ Factors } & \multirow{2}{*}{\multicolumn{2}{|c|}{$\begin{array}{c}\text { Mean (Standard Deviation) } \\
\text { Ownership of capital }\end{array}$}} & \multirow{2}{*}{\multicolumn{2}{|c|}{ t-test }} & \multirow{2}{*}{\multicolumn{3}{|c|}{$M-V U$ test }} \\
\hline & & & & & & & & \\
\hline & & $\mathrm{D}, \mathrm{N}=117$ & $F, N=61$ & $t$ & $\mathbf{p}^{*}$ & $U$ & $z$ & $\mathbf{p}^{*}$ \\
\hline \multirow{11}{*}{$\begin{array}{l}\text { Firm's } \\
\text { internal } \\
\text { environment }\end{array}$} & f1 & $2.29(1.130)$ & $2.15(1.108)$ & 0.807 & 0.421 & 3305.500 & -0.838 & 0.402 \\
\hline & f2 & $3.50(1.119)$ & $3.08(1.269)$ & 2.281 & 0.024 & 2880.000 & -2.184 & 0.029 \\
\hline & $\mathrm{f3}$ & $1.87(0.933)$ & $1.70(0.955)$ & 1.123 & 0.263 & 3152.500 & -1.375 & 0.169 \\
\hline & f4 & $2.03(1.156)$ & $1.62(0.840)$ & 2.656 & 0.009 & 2928.000 & -2.114 & 0.034 \\
\hline & f5 & $3.02(1.034)$ & $2.51(1.090)$ & 3.060 & 0.003 & 2693.000 & -2.799 & 0.005 \\
\hline & f6 & $2.55(1.118)$ & $2.30(1.038)$ & 1.462 & 0.146 & 3109.000 & -1.459 & 0.145 \\
\hline & f7 & $2.11(1.073)$ & $2.02(1.072)$ & 0.559 & 0.577 & 3382.000 & -0.601 & 0.548 \\
\hline & f8 & $2.62(0.990)$ & $2.61(1.159)$ & 0.053 & 0.958 & 3490.000 & -0.251 & 0.802 \\
\hline & f9 & $3.19(1.017)$ & $2.93(1.276)$ & 1.345 & 0.182 & 3207.000 & -1.156 & 0.248 \\
\hline & f10 & $2.40(1.175)$ & $2.31(1.205)$ & 0.482 & 0.630 & 3390.500 & -0.564 & 0.573 \\
\hline & f11 & $3.11(1.237)$ & $2.46(1.177)$ & 3.393 & 0.001 & 2557.000 & -3.187 & 0.001 \\
\hline \multirow{6}{*}{$\begin{array}{l}\text { Domestic } \\
\text { business } \\
\text { environment }\end{array}$} & $\mathrm{f} 12$ & $3.94(0.893)$ & $3.85(0.771)$ & 0.651 & 0.516 & 3252.500 & -1.029 & 0.304 \\
\hline & f13 & $3.55(1.126)$ & $3.54(0.959)$ & 0.036 & 0.972 & 3479.000 & -0.289 & 0.773 \\
\hline & f14 & $3.36(0.905)$ & $3.13(0.785)$ & 1.743 & 0.084 & 3249.500 & -1.115 & 0.265 \\
\hline & $\mathrm{f} 15$ & $3.85(0.883)$ & $3.74(0.929)$ & 0.824 & 0.411 & 3382.000 & -0.603 & 0.546 \\
\hline & $\mathrm{f} 16$ & $3.79(0.084)$ & $3.48(0.086)$ & 2.658 & 0.009 & 2807.000 & -2.520 & 0.012 \\
\hline & $\mathrm{f} 17$ & $3.32(0.075)$ & $3.23(0.095)$ & 0.694 & 0.488 & 3283.500 & -1.031 & 0.303 \\
\hline \multirow{6}{*}{$\begin{array}{l}\text { Foreign } \\
\text { markets }\end{array}$} & f18 & $4.10(1.062)$ & $4.07(1.078)$ & 0.219 & 0.827 & 3483.000 & -0.280 & 0.779 \\
\hline & f19 & $2.74(1.108)$ & 2.61 (1.069) & 0.793 & 0.429 & 3349.500 & -0.698 & 0.485 \\
\hline & $\mathrm{f} 20$ & $2.72(1.121)$ & $2.62(1.143)$ & 0.533 & 0.595 & 3394.000 & -0.557 & 0.577 \\
\hline & f21 & $2.27(0.906)$ & $1.90(1.028)$ & 2.480 & 0.014 & 2741.000 & -2.660 & 0.008 \\
\hline & f22 & $1.67(0.910)$ & $1.51(0.829)$ & 1.136 & 0.257 & 3224.000 & -1.202 & 0.230 \\
\hline & f23 & $2.48(1.103)$ & $2.08(1.069)$ & 2.301 & 0.023 & 2792.000 & -2.474 & 0.013 \\
\hline
\end{tabular}

* Statistically significant at $p<.05$

Source: authors' research 
The ANOVA and K-W test results, whose results are shown in Table 6, have not been identified the existence of statistically significant differences in estimation of investigation factors depending on the type of industry.

Table 6

\section{Differences in Evaluation of Investigated Factors by the Firms‘ Type of Industry}

\begin{tabular}{|c|c|c|c|c|c|c|c|c|c|}
\hline \multirow{3}{*}{$\begin{array}{l}\text { Environ- } \\
\text { ment }\end{array}$} & \multirow{3}{*}{ Factors } & \multicolumn{4}{|c|}{ Mean (Standard Deviation) } & \multirow{2}{*}{\multicolumn{2}{|c|}{ ANOVA }} & \multirow{2}{*}{\multicolumn{2}{|c|}{ K-W test }} \\
\hline & & \multicolumn{4}{|c|}{ Type of industry } & & & & \\
\hline & & LTI, $N=84$ & MLTI, N = 58 & MHTI, $\mathbf{N}=30$ & HTI, $N=6$ & $\mathbf{F}$ & $\mathbf{p}^{*}$ & $x^{2}$ & $\mathbf{p}^{*}$ \\
\hline \multirow{11}{*}{$\begin{array}{l}\text { Firm's } \\
\text { internal } \\
\text { environ- } \\
\text { ment }\end{array}$} & f1 & $2.11(1.064)$ & $2.38(1.182)$ & $2.30(1.179)$ & $2.50(1.049)$ & 0.825 & 0.482 & 2.453 & 0.484 \\
\hline & f2 & $3.27(1.196)$ & $3.40(1.242)$ & $3.40(1.133)$ & $4.00(0.632)$ & 0.757 & 0.520 & 2.472 & 0.480 \\
\hline & $\mathrm{f} 3$ & $1.86(0.971)$ & $1.79(0.951)$ & $1.63(0.850)$ & $2.33(0.816)$ & 1.046 & 0.374 & 3.951 & 0.267 \\
\hline & $\mathrm{f} 4$ & $1.87(1.027)$ & $1.86(1.067)$ & $1.80(1.095)$ & $2.83(1.472)$ & 1.658 & 0.178 & 3.893 & 0.273 \\
\hline & f5 & $2.81(1.081)$ & $2.86(1.099)$ & $2.83(1.053)$ & $3.17(1.169)$ & 0.211 & 0.088 & 0.432 & 0.934 \\
\hline & f6 & $2.40(1.121)$ & $2.57(1.061)$ & $2.27(1.048)$ & $3.17(1.169)$ & 1.417 & 0.239 & 3.699 & 0.296 \\
\hline & f7 & $1.94(1.034)$ & $2.33(1.082)$ & $1.90(0.923)$ & $2.50(1.761)$ & 2.140 & 0.097 & 5.614 & 0.132 \\
\hline & f8 & $2.62(1.040)$ & 2.60 (1.154) & $2.60(0.968)$ & $2.67(0.516)$ & 0.009 & 0.999 & 0.139 & 0.987 \\
\hline & f9 & $3.05(1.140)$ & $3.12(1.125)$ & $3.20(1.126)$ & $3.17(0.753)$ & 0.154 & 0.927 & 0.469 & 0.926 \\
\hline & $\mathrm{f} 10$ & $2.13(1.073)$ & $2.60(1.256)$ & $2.57(1.278)$ & $2.50(1.049)$ & 2.245 & 0.085 & 6.100 & 0.107 \\
\hline & f11 & $2.71(1.402)$ & 3.05 (1.033) & $2.93(1.172)$ & $3.50(1.225)$ & 1.368 & 0.254 & 4.510 & 0.211 \\
\hline \multirow{6}{*}{$\begin{array}{l}\text { Domestic } \\
\text { business } \\
\text { environ- } \\
\text { ment }\end{array}$} & f12 & $3.89(0.892)$ & $3.83(0.901)$ & $4.13(0.681)$ & $3.83(0.408)$ & 0.893 & 0.446 & 2.530 & 0.470 \\
\hline & f13 & $3.52(1.144)$ & $3.67(1.130)$ & $3.43(1.006)$ & $3.17(0.753)$ & 0.642 & 0.589 & 2.767 & 0.429 \\
\hline & f14 & $3.14(0.933)$ & $3.43(0.861)$ & $3.37(0.718)$ & $3.33(0.516)$ & 1.395 & 0.246 & 5.760 & 0.124 \\
\hline & f15 & $3.76(0.939)$ & $3.90(0.872)$ & $3.83(0.913)$ & $3.67(0.516)$ & 0.312 & 0.817 & 0.911 & 0.823 \\
\hline & f16 & $3.79(0.865)$ & $3.60(0.857)$ & $3.67(0.802)$ & $3.17(0.408)$ & 1.343 & 0.262 & 4.379 & 0.223 \\
\hline & $\mathrm{f} 17$ & $3.37(0.757)$ & $3.26(0.870)$ & $3.17(0.747)$ & $3.00(0.632)$ & 0.821 & 0.484 & 2.978 & 0.395 \\
\hline \multirow{6}{*}{$\begin{array}{l}\text { Foreign } \\
\text { markets }\end{array}$} & f18 & $4.08(1.020)$ & 4.09 (1.097) & $4.17(1.053)$ & $3.83(1.602)$ & 0.167 & 0.918 & 0.204 & 0.977 \\
\hline & f19 & $2.62(1.063)$ & $2.76(1.129)$ & $2.77(1.194)$ & $2.83(0.753)$ & 0.272 & 0.846 & 0.723 & 0.868 \\
\hline & $\mathrm{f} 20$ & $2.58(1.044)$ & $2.67(1.176)$ & $2.87(1.252)$ & $3.33(1.033)$ & 1.157 & 0.328 & 3.693 & 0.297 \\
\hline & f21 & $2.25(0.890)$ & $2.02(1.068)$ & $2.03(0.928)$ & $2.50(1.049)$ & 1.080 & 0.359 & 4.732 & 0.193 \\
\hline & $\mathrm{f} 22$ & $1.64(0.914)$ & $1.53(0.863)$ & $1.63(0.850)$ & $1.83(0.983)$ & 0.310 & 0.818 & 1.149 & 0.765 \\
\hline & f23 & $2.36(1.105)$ & $2.34(1.101)$ & $2.23(1.104)$ & $2.67(1.366)$ & 0.271 & 0.846 & 0.671 & 0.880 \\
\hline
\end{tabular}

* Statistically significant at $p<.05$

Source: authors' research

\section{Correlation analysis}

The Spearman's rank correlation, whose indicators are shown in Table 7, reveals the existence of the correlations between:

1) Firm's size and four factors: lack of capital for financing exports, a negative weak correlation; procedures and costs of loan for financing exports, a negative weak correlation; domestic currency exchange rate, a negative weak correlation, and association of exporters a weak negative correlation. That means that along with the firm's size the impact of these factors becomes weaker as a barrier to exports; 
2) Length of the export experience and four factors: ability to adjust the product to foreign market standards a positive weak correlation; provision of price competitiveness at foreign markets a positive weak correlation; export tariffs at foreign markets a positive weak correlation, and legal regulation at foreign markets a positive weak correlation. The stipulated factors have more impact on the more experienced exporters in terms of barriers to export;

Table 7

\section{Correlation of the Firms' Characteristics and the Investigated Factors}

\begin{tabular}{|c|c|c|c|c|c|c|c|c|c|}
\hline \multirow{2}{*}{ Environment } & \multirow[t]{2}{*}{$\begin{array}{l}\text { Fac- } \\
\text { tors }\end{array}$} & \multicolumn{2}{|c|}{$\begin{array}{c}\text { Firm's } \\
\text { size }\end{array}$} & \multicolumn{2}{|c|}{$\begin{array}{l}\text { Length of the } \\
\text { export experience }\end{array}$} & \multicolumn{2}{|c|}{$\begin{array}{l}\text { Ownership of } \\
\text { capital }\end{array}$} & \multicolumn{2}{|c|}{ Type of industry } \\
\hline & & $r s$ & $p$ & $r s$ & $p$ & $r s$ & $p$ & $r s$ & $p$ \\
\hline \multirow{11}{*}{$\begin{array}{l}\text { Firm } \\
\text { internal } \\
\text { environment }\end{array}$} & f1 & -0.064 & 0.395 & 0.082 & 0.276 & -0.063 & 0.404 & -0.034 & 0.649 \\
\hline & $f_{2}$ & $-0.179^{*}$ & 0.017 & 0.060 & 0.428 & $-0.164^{*}$ & 0.029 & 0.001 & 0.984 \\
\hline & f3 & -0.044 & 0.558 & 0.070 & 0.355 & -0.103 & 0.170 & -0.009 & 0.910 \\
\hline & f4 & -0.070 & 0.351 & -0.052 & 0.492 & $-0.159^{*}$ & 0.034 & 0.012 & 0.878 \\
\hline & $f_{5}$ & 0.012 & 0.874 & 0.121 & 0.109 & $-0.210^{* *}$ & 0.005 & 0.003 & 0.991 \\
\hline & $f_{6}$ & -0.134 & 0.075 & 0.010 & 0.891 & -0.110 & 0.145 & -0.068 & 0.367 \\
\hline & $f_{7}$ & -0.111 & 0.141 & -0.068 & 0.370 & -0.045 & 0.549 & -0.144 & 0.056 \\
\hline & f8 & 0.078 & 0.300 & $0.171^{*}$ & 0.022 & -0.019 & 0.802 & 0.006 & 0.933 \\
\hline & f9 & 0.109 & 0.147 & $0.224^{\star *}$ & 0.003 & -0.087 & 0.249 & 0.009 & 0.902 \\
\hline & $f 10$ & -0.025 & 0.739 & 0.093 & 0.219 & -0.042 & 0.574 & -0.050 & 0.506 \\
\hline & $f_{11}$ & 0.053 & 0.483 & 0.142 & 0.060 & $-0.240^{* *}$ & 0.001 & -0.044 & 0.556 \\
\hline \multirow{6}{*}{$\begin{array}{l}\text { Domestic } \\
\text { business } \\
\text { environment }\end{array}$} & $f_{12}$ & $-0.175^{*}$ & 0.020 & -0.007 & 0.928 & -0.077 & 0.305 & 0.098 & 0.191 \\
\hline & $f 13$ & -0.095 & 0.208 & 0.013 & 0.866 & -0.022 & 0.773 & -0.108 & 0.150 \\
\hline & f14 & -0.067 & 0.375 & 0.049 & 0.515 & -0.084 & 0.266 & -0.064 & 0.399 \\
\hline & $f 15$ & $-0.200^{* *}$ & 0.007 & 0.082 & 0.278 & -0.045 & 0.548 & -0.052 & 0.493 \\
\hline & f16 & -0.072 & 0.343 & 0.048 & 0.529 & $-0.189^{*}$ & 0.011 & 0.015 & 0.842 \\
\hline & $f_{17}$ & $-0.178^{*}$ & 0.017 & -0.099 & 0.188 & -0.077 & 0.304 & 0.012 & 0.879 \\
\hline \multirow[t]{6}{*}{ Foreign markets } & $f 18$ & 0.096 & 0.201 & 0.100 & 0.185 & -0.021 & 0.780 & 0.011 & 0.889 \\
\hline & f19 & 0.069 & 0.363 & $0.148^{*}$ & 0.049 & -0.052 & 0.487 & -0.002 & 0.982 \\
\hline & $\mathrm{f} 20$ & 0.121 & 0.109 & $0.175^{*}$ & 0.020 & -0.042 & 0.579 & 0.068 & 0.368 \\
\hline & $f 21$ & 0.086 & 0.256 & 0.844 & 0.494 & $-0.200^{* *}$ & 0.007 & 0.070 & 0.355 \\
\hline & $f_{22}$ & -0.036 & 0.636 & 0.015 & 0.844 & -0.090 & 0.231 & 0.068 & 0.368 \\
\hline & $f 23$ & 0.033 & 0.663 & 0.045 & 0.549 & $-0.186^{*}$ & 0.013 & -0.023 & 0.763 \\
\hline
\end{tabular}

* Statistically significant at $p<.05$

** Statistically significant at $p<.01$

Source: authors' research

3) Capital ownership and seven factors: lack of capital for financing exports a negative weak correlation; management commitment to export business a negative weak correlation; collecting information on foreign markets a negative weak correlation; promotion on foreign markets a negative weak correlation; national export branding policy a negative weak correlation; cooperation with foreign partners a negative 
weak correlation, and money inflow from the sale at foreign markets a negative weak correlation. That means that firms with foreign capital are exposed to smaller impact of the stipulated factors in terms of barriers to export.

The correlation has not been identified between the type of industry, which a firm belongs to, and investigated factors which can pose barriers in export.

\section{Discussion}

The results of the research show that Serbian exporters face barriers in 14 out of 23 investigated factors of the firm internal environment, domestic business environment and foreign markets. Ranked according to theirs' average grades, export barriers are presented in Table 8.

Table 8

\section{Rank of Barriers Serbian Exporters Face}

\begin{tabular}{|l|l|l|}
\hline Export Barriers & Environment & Mean \\
\hline Competition on foreign markets & Foreign markets & 4.09 \\
\hline $\begin{array}{l}\text { The costs and procedures for obtaining loans for } \\
\text { financing export }\end{array}$ & Domestic business environment & 3.93 \\
\hline Level of domestic currency exchange rate & Domestic business environment & 3.81 \\
\hline Lack of national export branding policy & Domestic business environment & 3.69 \\
\hline Government policy toward exporters & Domestic business environment & 3.54 \\
\hline Lack of capital for financing exports & Firm's internal environment & 3.36 \\
\hline Support and expertise through the export associations & Domestic business environment & 3.29 \\
\hline Domestic bureaucratic export procedures & Domestic business environment & 3.28 \\
\hline Price competitiveness on foreign markets & Firm's internal environment & 3.10 \\
\hline Promotion on foreign markets & Firm's internal environment & 2.89 \\
\hline Collecting information about foreign markets & Firm's internal environment & 2.84 \\
\hline Export tariffs and costs of sales on foreign markets & Foreign markets & 2.70 \\
\hline Legal regulations on foreign markets & Foreign markets & 2.69 \\
\hline Adjustment of products with foreign markets demands & Firm's internal environment & 2.61 \\
\hline
\end{tabular}

Source: authors' research

According to the environment, in which they are made, the most of these barriers, six or $42.9 \%$, originate from the domestic business environment, five or $35.7 \%$ originate from the firm internal environment, and three or $21.4 \%$ originate from foreign markets.

The stipulated barriers particularly affect small firms. Even certain factors of the internal environment of the firm, i.e. the level of knowledge about foreign markets and knowledge of export incentives, as well as transport to foreign markets, which are not recognized as barriers by the medium and large firms, small treat as a barriers to their export. In addition, small firm have more problems with providing capital to finance 
exports, the domestic currency exchange rate, language barriers and cross-cultural differences at foreign markets. These findings are in accordance with several previous studies (Majocchi et al., 2005; Jauhari, 2007). But, small firms show a greater capacity compared to the large ones to cope with the challenges of price competitiveness and to adjust their products to standards of foreign markets, foreign legal regulation is less complicated for them, and they have fewer problems regarding co-operation with foreign partners and the influx of money from sales at foreign markets. Based on the above, the Hypotheses 1 in case of Serbian exporters can only be partly confirmed.

Having analysed only the exporters with the longest and the shortest experience, the research shows that the exporters with less experience have smaller problems in terms of production capacities for exports, self-financing of exports, organization of export sector, management commitment to exports, information collection and knowledge on foreign markets as well as adjusting marketing mix factors to export in relation to exporters with the longest experience. The only element of the firm internal environment, which gives advantage to the exporters with the longest experience, is the possession of knowledge on export incentives. In terms of foreign markets factors, there are statistically significant differences in estimation of the amount of export tariffs and legal regulations at foreign markets, but these factors represent smaller barriers for exporters with the shortest experience. Based on these findings, in the case of Serbian exporters, Hypotheses 2 can be rejected. According to this finding, the results of the research are in compliance with the findings of several previous surveys (Moen and Servais, 2002; Lefebvre and Lefebvre, 2001).

The impact of all investigated factors in all three environments is higher in firms with domestic capital in relation to firms in which foreign capital is present. Statistically significant differences between these two groups of firms exist in the impact of the lack of capital for export financing, management commitment to export, collecting information and promotion at foreign markets within the range of the factors of the firm internal environment, the lack of national export branding policies within the factors of domestic business environment, and the cooperation with foreign partners and the influx of money from sales at foreign markets within the foreign market factors. Based on these findings in the case of Serbian exporters the Hypotheses 3 can be confirmed. According to this, the results of the research are in compliance with several previous surveys (Cole et al., 2010; Filatotchev et al., 2008).

Almost negligible differences in the assessment of all investigated factors between firms classified by the type of industry, none of which is within the scope of statistical significance, and the lack of correlation between the type of industry and the impact of the investigated factors, in the case of Serbian exporters give grounds for rejection of the Hypotheses 4. According to this, the results of the research are in compliance with several previous surveys (Gao et al., 2010; Lefebrve and Lefebrve, 2001). 
But, for more clearly interpretation of research results it need to be understood than Serbian exporters operate in specific environment, and compare this situation with one in EU Member Country. Czech Republic seems to be good example. Czech economy is one of the most open in the world, with a dominant export orientation and with very strong export support. Unlike Czech, Serbian economy is partially opened and import depended. Other export related data of Serbia and Czech Republic are shown in Table 9.

Table 9

Serbia and Czech Republic Export in 2011

\begin{tabular}{|l|l|l|}
\hline & Serbia & Czech Republic \\
\hline Export volume & 19.862 million USD & 162.339 million USD \\
\hline Export per capita & 2.038 USD & 14.904 USD \\
\hline Export share in GDP & $36.60 \%$ & $72.40 \%$ \\
\hline Growth rate of export & $15.40 \%$ & $17.20 \%$ \\
\hline $\begin{array}{l}\text { Main export products by } \\
\text { export share }\end{array}$ & $\begin{array}{l}\text { iron and steel, non-ferrous } \\
\text { metals, cereals, fruits and } \\
\text { vegetables }\end{array}$ & $\begin{array}{l}\text { machinery and transport } \\
\text { equipment, raw materials } \\
\text { and fuel, chemicals }\end{array}$ \\
\hline
\end{tabular}

Source: Serbia and Czech Republic national statistic yearbooks, 2012

The World Bank publication Doing Business in a More Transparent World 2011 ranked by easy for business the Czech Republic on 64, and Serbia on 92 position (The World Bank, 2012, p. 91 and p. 124). Besides that, exporters in Serbia and the Czech Republic operate in different domestic business environments. Also their competitiveness is very low. Some indicators from The Global Competitiveness Report 2011-2012 presented on Figure 2 may be good illustration.

Also, Serbia and the Czech Republic have different export support systems. In the Czech Republic it is mainly created through a joint efforts of the Czech Export Bank (CEB), Export Guarantee and Insurance Company (EGAIC). Export support commitments of CEB and EGAIC raised in recent years from 3.11 billion in 2008 to 5.03 billion USD in 2010 (Roteckeri, 2012). In 2011 funds made available to exporters by CEB grew up 20.5\% comparing to the previous year (Czech Export Bank, 2012, p.30) and EGAIC insured export credits, bank guarantees and investments abroad in the total volume about 3 billion USD (Export Guarantee and Insurance Company, 2012, p.5). These CEB and EGAIC activities were a significant factor that influences positively the volume of exports in the Czech Republic in 2011 (Janda et al., 2012). 


\section{Figure 2}

Competitiveness of Firms and Domestic Business Environment in Czech Republic and Serbia

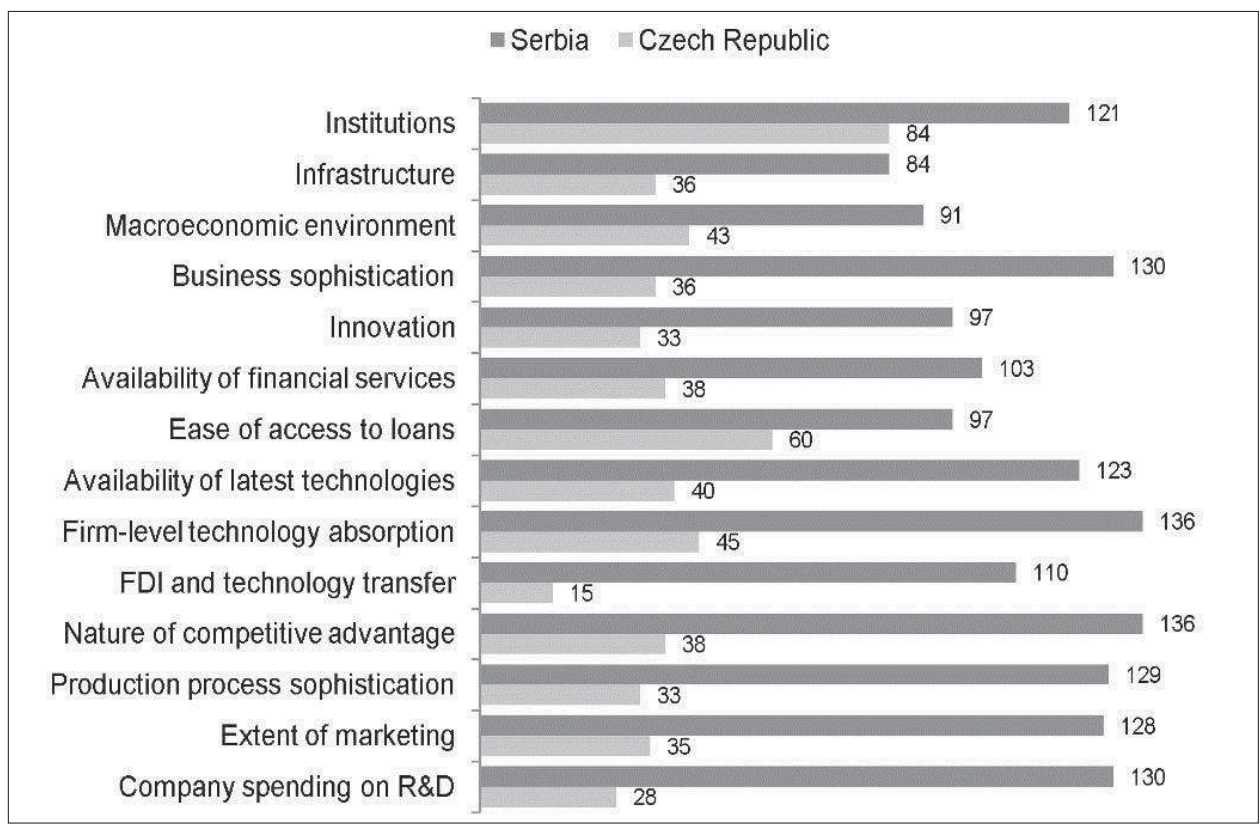

Source: The Global Competitiveness Report 2011-2012, World Economic Forum, Geneva, Switzerland, 2011, pp. 160-161 and 314-315.

Serbian system for export support consists of Serbian Investment and Export Promotion Agency (SIEPA) and Export Credit and Insurance Agency (ECIA). For export competitiveness increase programs and export promotion SIEPA invest 1.73 million USD in 2011 (SIEPA, 2012). ECIA approved 150 loans to Serbian exporters in total value of 90.35 million USD and issued guarantees worth 3.47 million USD in 2011 (ECIA, 2012). Serbian export supporting system is focused on export to EU and the neighbouring countries, but there were no special incentives to support exporting products of higher processing levels and products with greater foreign exchange rate effects, as well to support the development of exporters' innovation, although the majority of Serbian exports are raw materials and low level processing products. Also, the amount of export credit, issuance and guarantees in Serbia were very small and mostly on shortterm oriented, if we compare it with comparable one in Czech Republic.

\section{Conclusion}

The main objective of this study was to investigate the impact of the firm's size, the length of export experience, ownership of capital and type of industry which firm belongs to on perception of the factors of firm internal environment, domestic business environment and foreign markets which can create barriers to export. Based on the research 
findings in the case of Serbian exporters the impact of firm size is mixed, i.e. the impact of most of the investigated factors on the export of small firms is bigger, but there are factors whose influence on the firms is not so strong, compared to large ones. The length of export experience is usually inversely proportional to the perception of the investigated factors, i.e. the impact of the investigated factors is less in the firms with shorter experience if compared to their impact on the firms with the longest experience. The presence of foreign capital in firm positively affects export, but in terms of the types of industry it has been found out that there is no significant impact of that feature on the perception of the investigated factors. Most obstacles to export, over 75\%, is within the firm internal environment factors and domestic business environment, and the order of the average score that the respondents gave to the influence of barriers mainly coincides with findings in other developing countries.

From the findings of this study several implications can be drawn for the exporters' management, the economic policy makers and for possible future researches. The exporters' management has to find out what could be done to increase competitiveness. Special attention should be paid to improving the quality of products, their certification, design, innovation, implementing new technologies, greater investment in research and development, building competitive strategies for doing business at foreign markets and at international marketing. Also, special attention must be paid to exporters associating in clusters, because international practice shows that it is a successful way to overcome barriers in export.

However, the efforts of exporters in improving the firm internal environment can be unsuccessful if the economic policy makers do not take measures to improve the domestic business environment. In this sense, the debate should be open on the impact of exchange rate of domestic currency on export, which most threatens small firms. Also, measures which can help exporters and which may contribute to increased export should be considered in addition to the existing ones. Exporters associations should be supported, as well as clusters and certification of domestic products. Implementation of new technologies should be encouraged, as well as research and development. Also, additional consultancy services should be provided to the exporters. Key export sectors should be determined and the special attention should be drawn to them as well as to branding export. In addition, in the field of export crediting, by the example of the Czech Republic and some other countries, the export bank should be established for better support of domestic export.

The results of the research, although conducted in the case of exporters in one small and underdeveloped economy, can be used for future researches on this subject in other countries as a guideline for the development of research instruments as well as in comparative analysis. In addition, future researches may be supplemented by qualitative approach for the detection of factors that may affect exports, which were not investigated in this study, and for a deeper understanding of the problems in export and possible ways of surpassing them based on the examples of successful exporters. 
The findings in this paper should be interpreted in the light of several limitations and remarks. Firstly, research findings have been based on subjective assessments of exporters and should be likewise interpreted. This refers to, in particular, firm internal environment factors and ability of respondents to give an objective evaluation of their own strengths and weaknesses. Secondly, these conclusions have been drawn on the basis of the Serbian exporters, with an export income over a million dollar in 2011. These exporters are considered to be successful firms in the Serbian framework, hence one may assume that disclosed statistically significant differences among different groups of firms in evaluation of researched factors, which are usually quite small, and correlation coefficients which are low, might have been much more relevant provided the research had been conducted on a random sample, with all exporters in Serbia included. However, despite this, authors believe that the results presented here point at a good direction, one should reconsider heading to, if one wishes to boost export of Serbia. Thirdly, the research comprises only a limited number of factors that may present barriers to export, hence future researches should expand this number, as well as a number of firm characteristics examined.

\section{REFERENCES}

Armstrong, J. S., Overton, T. S. (1977), "Estimating Non-response Bias in Mail Surveys." Journal of Marketing Research, Vol. 14, No. 3, pp. 396-402.

Arteaga-Ortiz, J., Fernandez-Ortiz, R. (2010), "Why Don't We Use the Same Export Barrier Measurement Scale? An Empirical Analysis in Small and Medium-Sized Enterprises." Journal of Small Business Management, Vol. 48, No. 3, pp. 395-420.

Cavusgil, S.T., Zou, S. (1994), "Marketing Strategy- Performance Relationship: An Investigation of the Empirical Link in Export Market Ventures." Journal of Marketing, Vol. 58, No. 1, pp. 1-21.

Czech Export Bank (2012), Annual report 2011. Prague: Czech Export Bank.

Cole, M., Elliott, R., Virakul, S. (2010), "Firm Heterogeneity, Origin of Ownership and Export Participation." The World Economy, Vol. 33, No. 2, pp. 264-291.

Czech Statistical Office (2012), Statistical Yearbook 2011 - Foreign Trade of the Czech Republic. Prague: Czech Statistical Office.

DeVellis, R. F. (2003), Scale Development: Theory and Applications. 2nd Ed. Thousand Oaks, CA: Sage Publications.

Dillman, D. A. (2007), Mail and Internet Surveys - The Tailored Design Method, 2nd Ed. Hoboken, New Jersey: John Wiley \& Sons.

Dominguez, L., V., Sequeira, C. G. (1993), "Determinants of LDC Exporters' Performance: A Crossnational Study." Journal of International Business Studies, Vol. 24, No.1, pp. 19-40.

Ellis, P. D. (2010), The Essential Guide to Effect Sizes: An Introduction to Statistical Power, Meta-Analysis and the Interpretation of Research Results. Cambridge: Cambridge University Press.

Export Credit and Insurance Agency of Republic of Serbia. (2012), Business Report for 2011. Uzice: Export Credit and Insurance Agency of Republic of Serbia (in Serbian language only).

Export Guarantee and Insurance Corporation (2012), Annual Report 2011, Prague: Export Guarantee and Insurance Corporation.

Filatotchev, I., Stephan, J., Jindra, B. (2008), "Ownership Structure, Strategic Controls and Export Intensity of Foreign-invested Firms in Transition Economies." Journal of International Business Studies, Vol. 39, No. 7, pp. 1133-1148. 
Foundation for the Advancement of Economics (2010), Serbian Post-Crisis Economic Growth and Development Model 2011-2020. Belgrade: Foundation for the Advancement of Economics (in Serbian language only).

Gao, G. Y., Murray, J. Y. M., Kotabe, M., Lu, J. (2010), "A Strategy Tripod Perspective on Export Behaviour: Evidence from Domestic and Foreign Firms Based in An Emerging Economy." Journal of International Business Studies, Vol. 41, No. 3, pp. 377-396.

Haahti, A., Madupu, V., Yavas, U., Babakus, E. (2005), "Cooperative Strategy, Knowledge Intensity and Export Performance of Small and Medium Sized Enterprises." Journal of World Business, Vol. 40, No. 2, pp. 124-138.

Jauhari, V. (2007), "Analysing Export Intensity of the Select Electronics Firms in India," International Journal of Innovation Management, Vol. 11, No. 3, pp. 379-396.

Jenkins, R. (1979), "The Export Performance of Multinational Corporations in Mexican Industry." Journal of Development Studies, Vol. 15, No. 3, pp. 89-105.

Karel J., Michaliková, E., Skuhrovec, J. (2012), "Credit Support for Export: Evidence from the Czech Republic." CERGE-EI Working Papers wp461, The Center for Economic Research and Graduate Education - Economic Institute, Prague.

Katsikeas, C. S., Morgan, R. E. (1994), "Differences in Perceptions of Exporting Problems Based on Firm Size and Export Market Experience." European Journal of Marketing, Vol. 28, No. 5, pp.17-35.

Kneller, R., Pisu, M. (2011), "Barriers to Exporting: What Are They and Who Do They Matter?", The World Economy, Vol. 34, No. 6, pp. 893-930.

Lages, L. F. (2000), "A Conceptual Framework of the Determinants of Export Performance: Reorganising Key Variables and Shifting Contingencies in Export Marketing." Journal of Global Marketing, Vol. 13, No. 3, pp. 29-51.

Lefebvre, E., Lefebrve, L. A. (2001), "Innovative Capabilities as Determinants of Export Performance and Behavior: Alongitudinal Study of Manufacturing SMEs." in Kleinknecht, A., Mohnen, P. ed., Innovation and Firm Performance. Econometric Explorations of Survey Data, Basingstoke, London: Palgrave, pp. 281-309.

Lopez-Rodríguez, J. L., García-Rodríguez, R. M. (2005), “Technology and Export Behaviour: A Resourcebased View Approach." International Business Review, Vol. 14, No. 5, pp. 539-557.

Majocchi, A., Bacchiocchi, E., Mayrhofer, U. (2005), "Firm Size, Business Experience and Export Intensity in SMEs: A Longitudinal Approach to Complex Relationships." International Business Review, Vol. 14, No. 6, pp. 719-738.

Moen, O., Servais, P. (2002), "Born Global or Gradual Global? Examining the Export Behavior of Small and Medium-sized Enterprises." Journal of International Marketing, Vol. 10, No. 3, pp. 49-72.

Neupert, K. E., Baughn, C. C., Dao, T. T. L. (2006), "SME Exporting Challenges in Transitional and Developed Economies." Journal of Small Business and Enterprise Development, Vol. 13, No. 4, pp. 535-45.

Pertovic, P. (2011), "Serbian Economic Grow After Crisis - Net Export, Competiviness and Control of Public Spending." Enterprises' Economica, Vol. 59, No. 1-2, pp. 21-33 (in Serbian language only).

Pla-Barber, J., Alegre, J. (2007), "Analysing the Link between Export Intensity, Innovation and Firm Size in a Science-based Industry." International Business Review, Vol.16, No. 3, pp. 275-293.

Ramaswami, S. N., Yang, Y. (1990), "Perceived Barriers to Exporting and Export Assistance Requirements", in Cavusgil, S., T., Czinkota, M., R., ed., International Perspectives on Trade Promotion and Assistance. Westport, CT: Quorum Books, pp.187-206.

Roteckeri, J. (2012), "Competitiveness of the Czech Official Export Support Model in View of Global Trends." in Němečková, I., Machek, O. ed., Trends in International Business - Proceedings of 3rd International Joint Conference on Business Strategies on Global Markets. Prague: Vysoká škola ekonomická, Nakladatelství Oeconomica, pp. 85-95.

Sharma, K. (2003), "Factors Determining India's Export Performance." Journal of Asian Economics, Vol. 14, No. 3, pp. 435-446.

SIEPA (2012), Annual Report 2011. Belgrade: Serbian Investment and Export Promotion Agency (in Serbian language only) 
Sousa, C. D. A., Martínez-Lopez, F. J., Coelho, F. (2008), "The Determinants of Export Performance: A Review of the Research in the Literature between 1998 and 2005." International Journal of Management Reviews, Vol. 10, No. 4, pp. 343-374.

Statistic Office of Republic of Serbia. (2012), Statistical Yearbook 2011. Belgrade: Statistic Office of Republic of Serbia.

Stoian, M. C., Rialp, A., Rialp, J. (2011), "Export Performance under the Microscope: A Glance through Spanish Lenses." International Business Review, Vol. 20, No. 2, pp. 117-135.

Suarez-Ortega, S. (2003), "Export Barriers: Insights from Small and Medium-sized Firms." International Small Business Journal, Vol. 21, No. 4, pp. 403-419.

Tesform, G., Lutz, C. (2006), "A Classification of Export Marketing Problems of Small and Medium Sized Manufacturing Firms in Developing Countries." International Journal of Emerging Markets, Vol. 1, No. 3, pp. 262-281.

The Wold Bank (2012), Doing Business In a More Transparent World. Washington: The Wold Bank.

The World Economic Forum (2011), The Global Competitiveness Report 2011-2012. Geneva: The World Economic Forum.

Weisberg, H. F. (2005), The Total Survey Error Approach - A Guide to the New Science of Survey Research. Chicago, Illinois: The University of Chicago Press.

Wheeler, C., Ibeh, K., Dimitratos, P. (2008), "UK Export Performance Research: Review and Implications." International Small Business Journal, Vol. 26, No. 2, pp. 207-239.

Zou, S., Stan, S. (1998), "The Determinants of Export Performance: A Review of the Empirical Literature between 1987 and 1997." International Marketing Review, Vol.15, No. 5, pp. 333-356. 